\title{
Correlated scattering corrections to eikonal few-body models
}

\author{
J. M. Brooke and J. A. Tostevin \\ Department of Physics, School of Physics and Chemistry, University of Surrey, Guildford, Surrey GU2 7XH, United Kingdom
}

(Received 2 June 2000; published 10 November 2000)

\begin{abstract}
A basic assumption which underpins recent applications of eikonal few-body models to nuclear scattering and nuclear reactions is that of the addition of the constituent scattering phases. We investigate the accuracy of this assumption in the case of the elastic scattering of ${ }^{8} \mathrm{He}$, treated as a five-constituent $(\alpha+4 n)$ system, from a light target nucleus at energies of 10's of MeV/nucleon. To do so we calculate Feshbach's correlated scattering or overlapping potential contributions to the eikonal model phase shifts in this many-body case. We find that even for ${ }^{8} \mathrm{He}$, with ten contributing pair-wise potential overlaps, these terms introduce corrections that are small in comparison with available experimental precision and also compared to those phase additive noneikonal corrections which arise from the use of an improved description of the phase shifts for each of the constituent-target subsystems within the few-body model.
\end{abstract}

PACS number(s): 24.10.-i, 21.60.Gx, 25.10.+s, 25.70.Bc

\section{INTRODUCTION}

Semiclassical treatments of scattering include the interference and diffraction effects neglected in purely classical descriptions. In such approaches to high-energy scattering, partial wave sums can be replaced by integrals over impact parameters. These representations are connected formally by use of expansions of the Legendre polynomials [1] and by replacing sums over discrete angular momenta by integrals. The treatment of light rays as straight lines has a long history in optics. The eikonal method is then the semiclassical analog of the van de Hulst extension of the Rayleigh-Gans scattering of light $[2,3]$. The eikonal approximation was first applied to the wave function for particle scattering by Molière [4] and by Fernbach et al. [5]. The best known and most successful application of this type was developed by Glauber [6]. Subsequently, eikonal methods have been used extensively in nuclear physics.

The eikonal approximation is that the phase shift introduced in the scattering can be calculated assuming the particle trajectory is approximated to a straight line path through the field of the target at the appropriate impact parameter. It has been applied, typically, when the wavelength of the projectile is small compared to the size of the system and the projectile energy exceeds the potential strength responsible for the scattering. In general, the eikonal approximation ceases to be valid at low energies or large scattering angles, since the assumption of a straight line trajectory is less applicable. Several analyses of the eikonal approximation, to understand its range of validity and to extend its predictive power to lower energies and/or larger angles, have been discussed [7]. These methods involve obtaining improved descriptions of the scattering phase. Other approaches maintain the eikonal phase shift but then attempt to include the effects of deviations from the eikonal straight line trajectory using an effective impact parameter, e.g., Ref. [8]. The latter have been used in particular for approximate treatments of the Coulomb deflection in heavy-ion scattering.

The application of eikonal methods to few-body projectiles was also first discussed by Glauber [6] for the (threebody) deuteron-nucleus system at high energy. More re- cently such models have been applied extensively at lower energies, typically less than $100 \mathrm{MeV} /$ nucleon, to study reactions of two- and three-body halo nuclei such as ${ }^{11} \mathrm{Be}$, ${ }^{11} \mathrm{Li}$, and ${ }^{14} \mathrm{Be}$, effective three- and four-body problems, e.g., Refs. [9-13], and also for ${ }^{8} \mathrm{He}$, an effective six-body problem [14]. Implicit in these approaches is the use of the adiabatic approximation, that the incident energy is sufficiently high compared with ground state and typical excitation energies, that the motions internal to the projectile can be frozen for the duration of the interaction [15]. Comparisons of adiabatic and nonadiabatic methods $[16,17]$ for two-body projectiles indicate that for elastic scattering this approximation is good at incident energies as low as $20 \mathrm{MeV} /$ nucleon. The projectile incident energies of most interest in this work, 40-100 MeV/nucleon, relevant to beams produced in fragmentation reactions, are somewhat higher. We are not yet in a position to be able to quantify the corrections to the adiabatic approximation for the ${ }^{8} \mathrm{He}$ system studied here. Nevertheless, the eikonal methods provide essentially the only practical method for quantitative investigations of effective few-body systems and so an understanding of their accuracy on different energy and angular regions is of considerable importance. The present work is a contribution to such a study.

At lower energies the basis of the few-body eikonal model is as follows. For each impact parameter $b$ of the center of mass of the projectile the few-body eikonal model calculation of the scattering amplitude involves the expectation value, over the projectile's ground state few-body density, of the product of the eikonal approximations to each constituent-target $S$ matrix. That is, it is assumed that the phase shift for the projectile-target system is the sum of the (eikonal) phase shifts due to each cluster interacting independently with the target. These interactions between the projectile constituents and the target are represented by complex optical potentials with the real part of the potential describing refraction of the incident wave and the imaginary part the absorption of flux from each two-body elastic channel. Recently it was shown that if, instead of the eikonal phase shifts, one uses the exact partial wave phase shifts continued to a continuous angular momentum variable, called the exact continued (EC) prescription, one improves the calculated 
cross sections at lower energies significantly [18-20]. One should note that, even with this replacement, it is still assumed that the total phase shift is the sum of the phase shifts generated by each constituent cluster with the target. It is implicit therefore that each two-body scattering takes place and is calculated without regard to the positions of the other constituents.

This approximation, which underpins recent applications, may not be accurate particularly when the number of constituents in the projectile increases and especially if these are localized in a small spatial volume. The target may then be under the influence of two cluster interactions simultaneously. This adds correlation terms in the total phase shift function that depend nonlinearly on the pairwise clustertarget interactions. Feshbach discusses and derives, for the many nucleon case, these overlapping potential contributions to the total phase shift within the semiclassical approximation [21-23].

The motivation for this paper is to assess the importance of these corrections to the eikonal model. We consider ${ }^{8} \mathrm{He}$ scattering from ${ }^{12} \mathrm{C}$. An earlier eikonal model analysis of this system, at $60 \mathrm{MeV} /$ nucleon [14], was in reasonable agreement with available quasielastic scattering data. The ${ }^{8} \mathrm{He}$ system has been chosen here, not due to these data, but because in ${ }^{8} \mathrm{He}$ the larger number of clusters (five) involved occupy a relatively smaller volume than is usual for halo nuclei, and thus those effects generated through the overlapping potentials should be emphasized for this system. Our use of the harmonic oscillator based cluster orbital shell model approximation (COSMA) for the ${ }^{8} \mathrm{He}$ wave function [24], with Gaussian rather than realistic single nucleon asymptotics, also confines the valence nucleons to smaller radii and will thus also tend to increase the importance of the overlapping potential contributions. We expect therefore that the calculations presented will provide an upper bound on the likely importance of such terms and an indication of the need for their treatment in other cases. They are expected to be significantly smaller for more weakly bound and more extended one- and two-neutron halo nuclei.

In the following, we discuss separately the magnitudes of corrections arising (1) from the leading overlapping potential terms, in the straight line trajectory limit and (2) from phase additive improvements to the cluster-target phase shifts due to nonstraight line trajectory modifications. We note that for high energy $(\approx 1 \mathrm{GeV})$ hadronic collisions a more unified treatment of noneikonal and several other corrections was made possible by use of a $1 / k$ perturbation expansion scheme by Wallace [25]. This expansion scheme is not readily applicable for the lower energy potential model description of interest here.

\section{FEW-BODY EIKONAL MODEL}

Assuming central two-body interactions, the $(n+1)$-body eikonal elastic scattering amplitude for the scattering of a $n$-body projectile, with ground state wave function $\phi_{0}^{(n)}$, from a target is [13]

$$
f_{0}^{(n+1)}(\theta)=-i k \int_{0}^{\infty} b d b J_{0}(q b)\left\{S_{0}^{(n)}(b)-1\right\},
$$

where $k$ is the projectile incident wave number in the center of mass frame, and $q=2 k \sin (\theta / 2)$ is the momentum transfer. The composite nature of the projectile is contained within $S_{0}^{(n)}(b)$, which is the eikonal approximation to the projectiletarget elastic $S$ matrix expressed as a function of impact parameter $b$,

$$
S_{0}^{(n)}(b)=\left\langle\phi_{0}^{(n)}\left|\prod_{j=1}^{n} S_{0}^{j}\left(b_{j}\right)\right| \phi_{0}^{(n)}\right\rangle .
$$

Here, each $S_{0}^{j}\left(b_{j}\right)=\exp \left[i \mathcal{X}_{0}^{j}\left(b_{j}\right)\right]$ is the eikonal $S$ matrix describing the interaction between cluster $j$ and the target at its own impact parameter $b_{j}$. The bra-ket notation in this equation implies integration over the projectile's internal coordinates. The eikonal $S$ matrices are related to the eikonal phase shift functions $\mathcal{X}_{0}^{j}\left(b_{j}\right)$, and hence to the two-body interactions $V_{j}$ with the target, through

$$
\mathcal{X}_{0}^{j}\left(b_{j}\right)=-\frac{\mu_{p t}}{\hbar^{2} k} \int_{-\infty}^{\infty} d z V_{j}\left(\sqrt{b_{j}^{2}+z^{2}}\right),
$$

where $\mu_{p t}$ is the projectile-target reduced mass. The treatment of Coulomb interactions within the $V_{j}$ is discussed in detail in Ref. [20] and leads to an analogous expression for the scattering amplitude

$$
\bar{f}_{0}^{(n+1)}(\theta)=f_{p t}(\theta)-i k \int_{0}^{\infty} b d b J_{0}(q b) e^{i \mathcal{X} p t(b)}\left\{\bar{S}_{0}^{(n)}(b)-1\right\},
$$

where $\bar{S}_{0}^{(n)}$ is now the eikonal elastic $S$ matrix, including Coulomb interactions, and $f_{p t}$ is the point charge Coulomb scattering amplitude. In the calculations presented here we do not include Coulomb breakup and so the Coulomb interaction is assumed to act on the ${ }^{8} \mathrm{He}$ center of mass. In this case the Coulomb modified projectile-target elastic $S$ matrix is

$$
\bar{S}_{0}^{(n)}(b)=S_{0}^{(n)}(b) \exp \left[i \mathcal{X}_{0 \rho}(b)-i \mathcal{X}_{p t}(b)\right],
$$

where $S_{0}^{(n)}$ is the (nuclear) $S$ matrix of Eq. (2) and the exponential factor accounts for the difference between the scattering phase of the actual Coulomb potential and that due to point charge scattering.

\section{NONEIKONAL PHASE CORRECTIONS}

Methods offering a framework with which to make systematic corrections to the eikonal phase have proved useful. Originally, the eikonal phase was replaced with the WentzelKramers-Brillouin (WKB) phase [26,27]. Subsequently Rosen and Yennie [28] developed this approach, as did Wallace [7,29-31]. Very recently, it was shown that using directly the exact partial wave phase shift, continued to a continuous angular momentum variable, the exact continued 


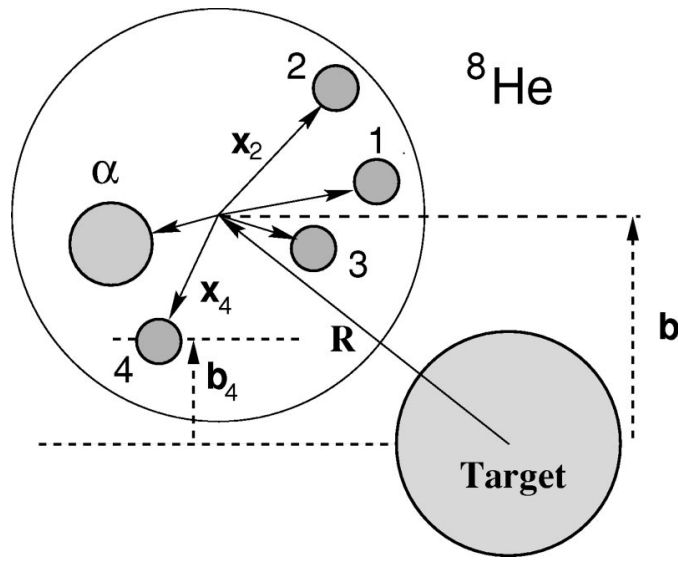

FIG. 1. Definition of the coordinate system used for the description of the effective six-body ${ }^{8} \mathrm{He}+$ target system.

(EC) prescription [20], provides a very much simpler procedure. Rather than develop and sum an expansion for the phase shift, one solves directly the radial Schrödinger equation at the required impact parameters $b_{j}$, and therefore for noninteger angular momenta $\lambda$. These $\lambda$ are associated with the physical angular momenta $l$, and $b_{j}$, according to $\lambda$ $=b_{j} k=\ell+1 / 2$. Each component $S$ matrix $S^{j}\left(b_{j}\right)$ is obtained by matching, in the normal manner, the numerical solutions of the radial equation for the potentials $V_{j}$ to the required asymptotic solutions, also analytically continued for real $\lambda$ [20].

These EC $S$ matrices, which include noneikonal corrections to each cluster-target phase shift to all orders, can be used in place of the $S_{0}^{j}$ in Eqs. (2) and (5). The resulting calculation retains the structure of the few-body eikonal model, involving a product of each cluster $S$ matrix and hence the assumption of addition of constituent phases. The inclusion of the leading order correlated scattering corrections are discussed in the next section.

\section{OVERLAPPING POTENTIAL CORRECTIONS}

In the semiclassical approximation of Feshbach [21-23], the derivation of the scattering amplitude develops in a similar fashion to that of the eikonal approximation. The coordinate system used is shown in Fig. 1. An expression for the scattering amplitude is developed which is similar to the few-body eikonal expression, except that higher order terms in the expansion of the exponential argument are retained. Feshbach writes

$$
\begin{aligned}
f_{F}^{(n+1)}(\theta)= & -i k \int_{0}^{\infty} b d b J_{0}(q b) \\
& \times\left[\left\langle\phi_{0}^{(n)}|\exp \{i \mathcal{X}(\boldsymbol{b},\{\boldsymbol{x}\})\}| \phi_{0}^{(n)}\right\rangle-1\right],
\end{aligned}
$$

where the phase function $\mathcal{X}$ is

$$
\mathcal{X}(\boldsymbol{b},\{\boldsymbol{x}\})=\int_{-\infty}^{\infty} d z \Phi(\boldsymbol{R},\{\boldsymbol{x}\})
$$

and $\{\boldsymbol{x}\}$ denotes the set of the $n$ constituents' position vectors relative to the projectile's center of mass. These satisfy $\sum_{j} m_{j} \boldsymbol{x}_{j}=0$ where $m_{j}$ is the mass of constituent $j$. The higher order terms are included in the differential phase function $\Phi$ [23] and, for a ${ }^{8} \mathrm{He}$ nucleus modeled as an alpha core and four neutrons, it can be written

$$
\Phi(\boldsymbol{R},\{\boldsymbol{x}\})=\left[k^{2}-\sum_{j} U_{j}\left(r_{j}\right)\right]^{1 / 2}-k,
$$

where $j=\alpha, n_{1}, \ldots, n_{4}$ and the $U_{j}$ are the reduced potentials $U_{j}(r)=2 \mu_{p t} V_{j}(r) / \hbar^{2}$. Defining a differential phase function for each constituent as

$$
\phi_{j}(r)=\left[k^{2}-U_{j}(r)\right]^{1 / 2}-k,
$$

rearranging Eq. (9) for $U_{j}$, substituting in Eq. (8), and using the binomial expansion to second order in the $\phi_{j}$ then gives

$$
\Phi(\boldsymbol{R},\{\boldsymbol{x}\})=\sum_{j} \phi_{j}\left(r_{j}\right)-\frac{1}{2 k} \sum_{j, m \neq j} \phi_{j}\left(r_{j}\right) \phi_{m}\left(r_{m}\right)+\cdots,
$$

and hence the phase shift function is

$$
\begin{aligned}
\mathcal{X}(\boldsymbol{b},\{\boldsymbol{x}\})= & -\frac{1}{2 k} \int_{-\infty}^{\infty} d z \sum_{j} U_{j}\left(r_{j}\right) \\
& -\frac{1}{8 k^{3}} \int_{-\infty}^{\infty} d z \sum_{j, m \neq j} U_{j}\left(r_{j}\right) U_{m}\left(r_{m}\right) .
\end{aligned}
$$

The first term of Eq. (11) is precisely the usual eikonal addition of phases representation $\mathcal{X}_{0}(\boldsymbol{b},\{\boldsymbol{x}\})=\Sigma_{j} \mathcal{X}_{0}^{j}\left(b_{j}\right)$. There are of course additional terms, of order $k^{-3}$ and higher, which involve the interaction $U_{j}$ of only a single constituent $j$. These are the noneikonal phase corrections, discussed in Sec. III, and which will be calculated to all orders.

The remaining terms, which we denote $\mathcal{X}_{F}$, are the leading Feshbach overlapping potential terms, i.e.,

$$
\mathcal{X}_{F}(\boldsymbol{b},\{\boldsymbol{x}\})=-\frac{1}{8 k^{3}} \int_{-\infty}^{\infty} d z \sum_{j, m \neq j} U_{j}\left(r_{j}\right) U_{m}\left(r_{m}\right) .
$$

Clearly, in the case of ${ }^{8} \mathrm{He}$, this is a sum of ten pair-wise terms. There will be contributions to $\mathcal{X}_{F}$ from those spatial configurations of the constituents in the projectile which, at a given impact parameter $b$ of its center of mass, can interact simultaneously with the target. The overlapping-potentialcorrected projectile-target elastic $S$ matrix is, finally,

$$
\begin{aligned}
S_{F}^{(n)}(b) & =\left\langle\phi_{0}^{(n)}\left|\exp \left[i \mathcal{X}_{0}(\boldsymbol{b},\{\boldsymbol{x}\})+\mathcal{X}_{F}(\boldsymbol{b},\{\boldsymbol{x}\})\right]\right| \phi_{0}^{(n)}\right\rangle \\
& =\left\langle\phi_{0}^{(n)}\left|\left\{\prod_{j=1}^{n} S_{0}^{j}\left(b_{j}\right)\right\} \exp \left[i \mathcal{X}_{F}(\boldsymbol{b},\{\boldsymbol{x}\})\right]\right| \phi_{0}^{(n)}\right\rangle .
\end{aligned}
$$

The same Coulomb phase is applied to this $S$ matrix as appears in Eq. (5). 


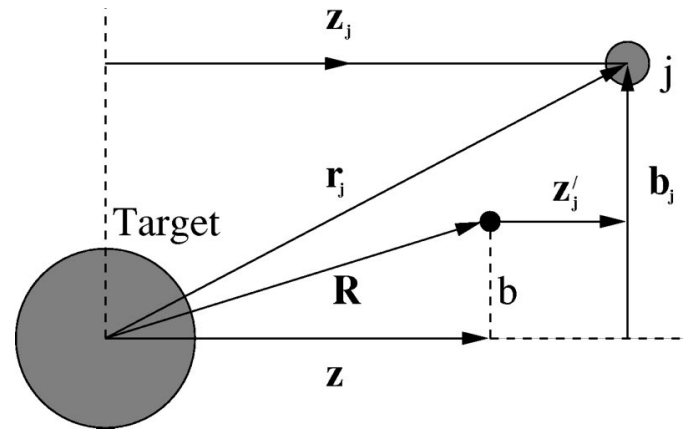

FIG. 2. Definition of the vector coordinates and components of a projectile constituent $j$ with respect to the target and with respect to the projectile's center-of-mass position.

\section{APPLICATION TO ${ }^{8} \mathrm{He}+{ }^{12} \mathrm{C}$ SCATTERING}

We apply the formalism above to the elastic scattering of ${ }^{8} \mathrm{He}$ from ${ }^{12} \mathrm{C}$ at $60 \mathrm{MeV} /$ nucleon. The 12 dimensional spatial integral, over four independent internal coordinates $\boldsymbol{x}_{j}$, involved in the calculation of the ${ }^{8} \mathrm{He}$ composite $S$ matrix, Eq. (13), is calculated by use of random sampling integration. Use is also made of the harmonic oscillator-based cluster orbital shell model approximation (COSMA) wave function for ${ }^{8} \mathrm{He}$ [24]. The details of this integration are the same as are presented in Ref. [14]. There, calculations are for the eikonal case, when Eq. (13) is evaluated assuming $\mathcal{X}_{F}=0$. Here the additional factor involving $\mathcal{X}_{F}$ must be included in the integrand. The explicit form of this term is now clarified.

\section{A. Feshbach phase}

Writing $\epsilon=\mu_{p t} /\left(\hbar^{2} k^{2}\right)$, Eq. (12) can be written

$$
\mathcal{X}_{F}(\boldsymbol{b},\{\boldsymbol{x}\})=-\frac{k \boldsymbol{\epsilon}^{2}}{2} \int_{-\infty}^{\infty} d z \sum_{j, m \neq j} V_{j}\left(r_{j}\right) V_{m}\left(r_{m}\right) .
$$

For simplicity, Gaussian neutron and $\alpha$ particle target potentials are used, i.e.,

$$
V_{j}(r)=\left(V_{0}^{j}+i W_{0}^{j}\right) \exp \left(-\gamma_{j} r^{2}\right)
$$

with $\gamma_{j}=1 / a_{j}^{2}$, and $a_{j}$ the potential range. Decomposing (see Fig. 2) each constituent position vector with respect to the target in terms of its impact parameter, $\boldsymbol{r}_{j}=\boldsymbol{b}_{j}+\boldsymbol{z}_{j}$ where $z_{j}$ $=z+z_{j}^{\prime}$, in Eq. (14), and defining

$$
\mathcal{V}\left(b_{j}, z_{j}^{\prime}\right)=\left(V_{0}^{j}+i W_{0}^{j}\right) \exp \left(-\gamma_{j}\left[b_{j}^{2}+z_{j}^{\prime 2}\right]\right),
$$

then

$$
\begin{aligned}
\mathcal{X}_{F}= & -\frac{k \epsilon^{2}}{2} \sum_{j, m \neq j} \mathcal{V}\left(b_{j}, z_{j}^{\prime}\right) \mathcal{V}\left(b_{m}, z_{m}^{\prime}\right) \int_{-\infty}^{\infty} d z \\
& \times \exp \left[-\left(\gamma_{j}+\gamma_{m}\right) z^{2}-2\left(\gamma_{j} z_{j}^{\prime}+\gamma_{m} z_{m}^{\prime}\right) z\right] .
\end{aligned}
$$

After integration over $z$, the Feshbach phase, for Gaussian potentials, is therefore

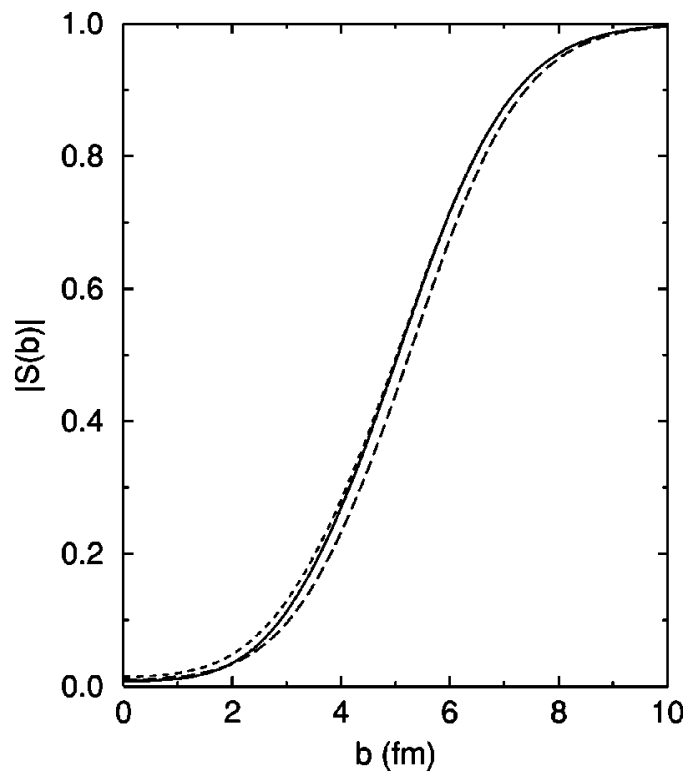

FIG. 3. Modulus of the calculated eikonal (solid line), the EC phases (long-dashed line), and the eikonal plus overlapping potential corrections (short-dashed line) elastic $S$ matrix, as a function of impact parameter, for ${ }^{8} \mathrm{He}+{ }^{12} \mathrm{C}$ scattering at $60 \mathrm{MeV} /$ nucleon.

$$
\begin{aligned}
\mathcal{X}_{F}(\boldsymbol{b},\{\boldsymbol{x}\})= & -\frac{k \boldsymbol{\epsilon}^{2}}{2} \sum_{j, m \neq j} \mathcal{V}\left(b_{j}, z_{j}^{\prime}\right) \mathcal{V}\left(b_{m}, z_{m}^{\prime}\right) \\
& \times\left(\frac{\pi}{\gamma_{j}+\gamma_{m}}\right)^{1 / 2} \exp \left[\frac{\left(\gamma_{j} z_{j}^{\prime}+\gamma_{m} z_{m}^{\prime}\right)^{2}}{\gamma_{j}+\gamma_{m}}\right] .
\end{aligned}
$$

\section{B. Numerical results}

The parameters of the Gaussian interactions are chosen to reproduce approximately the potentials used in the earlier eikonal analysis [14]. The $\alpha+{ }^{12} \mathrm{C}$ potential is $V_{0}^{\alpha}=-85.0$ $\mathrm{MeV}, W_{0}^{\alpha}=-28.0 \mathrm{MeV}, a_{\alpha}=3.0 \mathrm{fm}$, and the $n+{ }^{12} \mathrm{C}$ potential is $V_{0}^{n}=-37.4 \mathrm{MeV}, W_{0}^{n}=-10.0 \mathrm{MeV}, a_{n}=3.1 \mathrm{fm}$.

We first assess the nature of the Feshbach and EC phase corrections at the level of the ${ }^{8} \mathrm{He}$ elastic $S$ matrix. These are presented, as $|S(b)|$, in Fig. 3. The purely eikonal calculation is shown by the solid line. The short-dashed line shows the results when the Feshbach corrections $\mathcal{X}_{F}$ of Eq. (18) are included in Eq. (13). As might be expected, the correlated scattering corrections arise principally at relatively small impact parameters since it is along these trajectories that there are very significant simultaneous multiconstituent overlaps with the target. The fractional changes in $|S(b)|$ are large, of order $50 \%$ as clarified below, however, the constituents are also strongly absorbed along such paths. These overlapping potential corrections are seen to become very small for the grazing trajectories in the region of the nuclear surface. The long-dashed line in Fig. 3 shows the corresponding result when the noneikonal EC two-body $S$ matrices are used, instead of the $S_{0}^{j}\left(b_{j}\right)$, in Eq. (13). Now $\mathcal{X}_{F}=0$. This EC phase introduction shows significant modifications to the elastic $S$ matrix in the nuclear surface region. This is a direct consequence of the extended range of the exact (EC) two- 


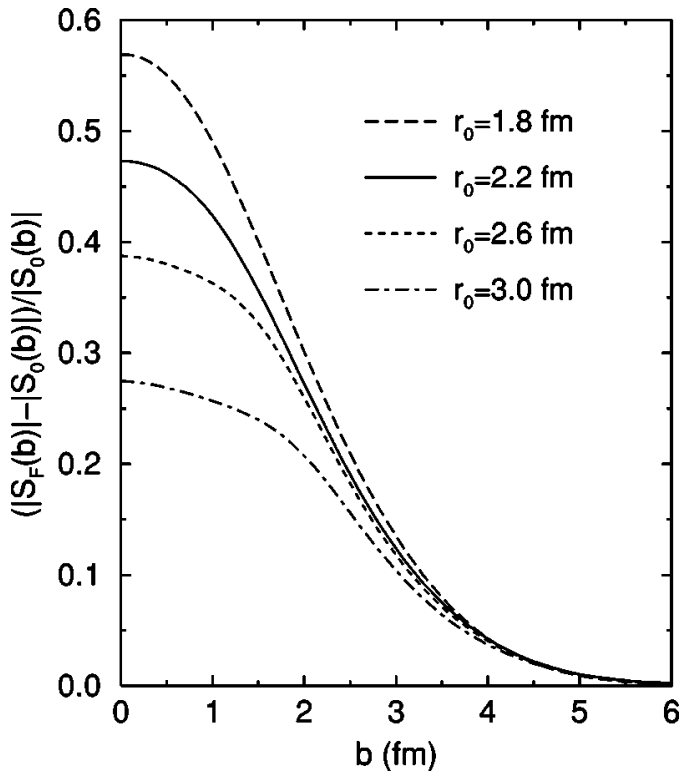

FIG. 4. Fractional changes of the moduli of the Feshbach modified and the eikonal $S$ matrices as a function of the assumed oscillator radius parameter $r_{0}$ in the COSMA wave function, for ${ }^{8} \mathrm{He}$ $+{ }^{12} \mathrm{C}$ scattering at $60 \mathrm{MeV} /$ nucleon.

body $S$ matrices with impact parameter compared to those of the eikonal limit, Eq. (3). These effects on the constituent $S$ matrices were shown in Figs. 1 and 3 of Ref. [20].

That the changes in $|S(b)|$, arising from $\mathcal{X}_{F}$, are large are shown in Fig. 4. This shows the fractional change in modulus of the Feshbach modified $\left|S_{F}(b)\right|$ from that of the eikonal calculation $\left(\left|S_{F}(b)\right|-\left|S_{0}(b)\right|\right) /\left|S_{0}(b)\right|$, as a function of the assumed oscillator radius parameter $r_{0}$ in the COSMA wave function. This value is usually taken as $2.2 \mathrm{fm}$ [24], based on the resulting rms size of ${ }^{8} \mathrm{He}$ for which the change is of order $50 \%$ in the target volume. Decreasing $r_{0}$ in the calculations further localizes the five constituents with an observed increase in the importance of the correlated scattering terms, to $57 \%$ at $b=0$ for $r_{0}=1.8 \mathrm{fm}$. In all cases, however, these effects are confined to small impact parameters of the projectile's center of mass.

The corresponding elastic scattering differential cross section angular distributions, presented as the ratio to Rutherford, are shown in Figs. 5 and 6 for $60 \mathrm{MeV} /$ nucleon incident energy and different angular ranges. The curves have the same meanings as in Fig. 3. As would be deduced from the detailed discussion of the $S$ matrices, the small impact parameter localization of overlapping potential corrections leads to significant modifications to the calculated cross sections only at larger center of mass scattering angles, Fig. 5. It should be pointed out that the quasielastic scattering data of Ref. [14], at $60 \mathrm{MeV} /$ nucleon, included center-of-mass scattering angles of less than $20^{\circ}$ and the error bars on those data are large compared to the effects calculated here. For this angular range, Fig. 6, the EC phase modifications are far more significant than the correlated scattering modifications. However, as the figure shows, and was also shown in Ref. [20], these noneikonal EC modifications are themselves already small at this energy, and smaller than the errors on the

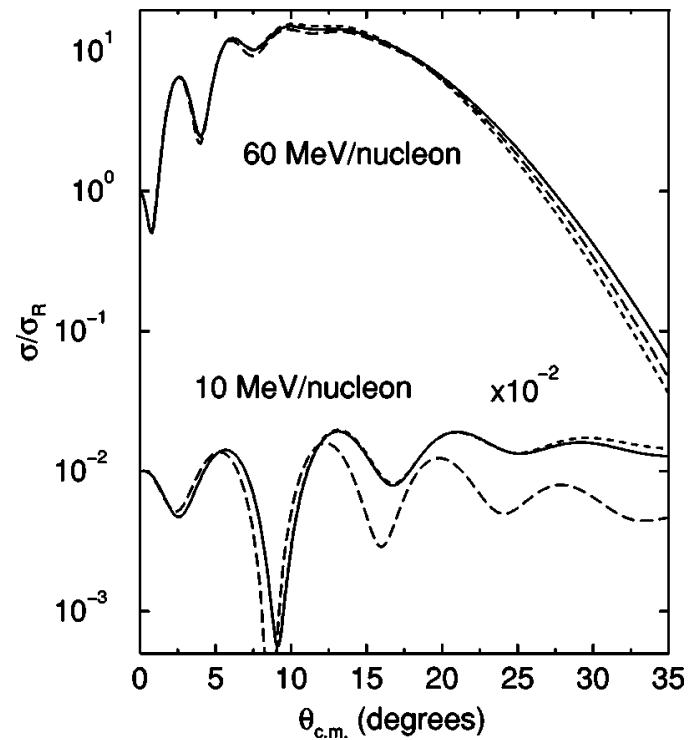

FIG. 5. Calculated ${ }^{8} \mathrm{He}+{ }^{12} \mathrm{C}$ elastic differential cross section angular distributions (as ratio to Rutherford) at 60 and $10 \mathrm{MeV} /$ nucleon. The curves show the results of the eikonal (solid line), the EC phases (long-dashed line), and the eikonal plus overlapping potentials (short-dashed line) calculations.

data available currently. Figure 5 therefore also shows calculations at an incident energy of $10 \mathrm{MeV} /$ nucleon. At this energy one would expect there to be additional corrections, due to our use of the adiabatic approximation. These calculations show nevertheless that, while the EC phase corrections become very significant, the overlapping potential effects remain very small upon calculated cross sections.

The sensitivity of these few-body model calculations to variations of physical parameters, such as the real and imaginary potential strengths, their range and diffuseness, and of the valence particle separation energy, has also been investigated for single neutron halo nuclei, such as ${ }^{11} \mathrm{Be}$, with fewer bodies and larger spatial extension. These can be found elsewhere [32]. There it is confirmed that, for such systems, the overlapping potential contributions are of even less significance.

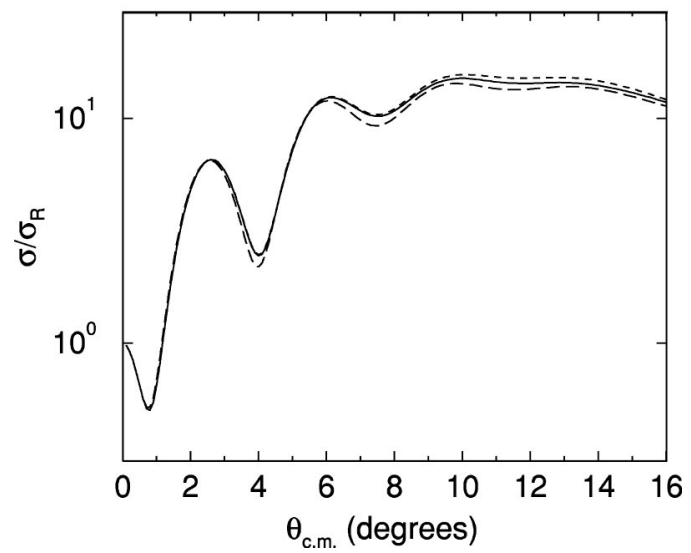

FIG. 6. As for Fig. 5, for small angles and for incident energy of $60 \mathrm{MeV} /$ nucleon. 


\section{SUMMARY AND CONCLUSIONS}

Coupled channels formulations have been developed which, within the few-body adiabatic approximation, allow for essentially exact calculations of the scattering of effective three-body projectiles [33]. Whereas, within eikonal methods, all break-up relative angular momenta between fragments are implicitly included, in the coupled channels approach these must be specified. The number of coupled channels increases very rapidly with the number of bodies and included angular momenta. As a result, eikonal and impact parameter based models currently provide the most practical methods for quantitative investigations of effective few-body systems, such as discussed here for ${ }^{8} \mathrm{He}$, and an assessment of the accuracy of these approximate calculations is of considerable importance.

As part of such an assessment, in this paper we have calculated the importance of Feshbach's correlated scattering or overlapping potential contributions to the eikonal model. The importance of these terms is also compared to those phase additive noneikonal corrections introduced when using an improved description of the phase shift for each of the constituent-target subsystems within the few-body model. ${ }^{8} \mathrm{He}$ was chosen because its larger number of clusters (five) occupy a smaller volume than is usual for halo nuclei. Thus the effects due to the overlapping potential terms should be emphasized for this system. We use the COSMA ${ }^{8} \mathrm{He}$ wave function, for simplicity, and obtain the expected sensitivity of the correlated scattering terms to the assumed size of the wave function. The use of the EC phases in the present sixbody, ${ }^{8} \mathrm{He}+{ }^{12} \mathrm{C}$, system is straightforward and the results are presented here at 60 and $10 \mathrm{MeV} /$ nucleon. We have shown that, even for the ${ }^{8} \mathrm{He}$ system, the small impact parameter localization of the corrections due to the overlapping potential terms means that their effects are very small on cross sections at center of mass angles currently accessible to experiment. Those noneikonal corrections due to use of the EC phases are more significant at center of mass scattering angles below $20^{\circ}$, and in particular at the lower energy. We conclude that at the incident energies of exotic beams produced in fragmentation reactions, the addition of phases assumption is remarkably accurate in few-body calculations on light target nuclei and that correlated scattering terms are unlikely to be significant in any application of the few-body models.

\section{ACKNOWLEDGMENTS}

The financial support of the Engineering and Physical Sciences Research Council (U.K.) through Grant Nos. GR/J 95867 and GR/M 82141 is gratefully acknowledged.
[1] H. M. MacDonald, Proc. London Math. Soc. 13, 220 (1914).

[2] R. G. Newton, Scattering Theory of Waves and Particles (McGraw-Hill, New York, 1966).

[3] H. C. van de Hulst, Light Scattering by Small Particles (Dover, New York, 1957).

[4] G. Molière, Z. Naturforsch. A 2, 133 (1947).

[5] S. Fernbach, R. Serber, and T. Taylor, Phys. Rev. 75, 1352 (1949)

[6] R.J. Glauber, in Lectures in Theoretical Physics, edited by W.E. Brittin (Interscience, New York, 1959), Vol. 1, p. 315.

[7] S. J. Wallace, Ann. Phys. (N.Y.) 78, 190 (1973).

[8] A. Vitturi and F. Zardi, Phys. Rev. C 36, 1404 (1987).

[9] G. F. Bertsch, K. Hencken, and H. Esbensen, Phys. Rev. C 57, 1366 (1998).

[10] A. Bonaccorso and D. M. Brink, Phys. Rev. C 58, 2864 (1998)

[11] C. A. Bertulani and H. Sagawa, Nucl. Phys. A588, 667 (1995).

[12] K. Yabana, Y. Ogawa, and Y. Suzuki, Phys. Rev. C 45, 2909 (1992); Nucl. Phys. A539, 295 (1992).

[13] J. S. Al-Khalili, I. J. Thompson, and J. A. Tostevin, Nucl. Phys. A581, 331 (1995).

[14] J. A. Tostevin, J. S. Al-Khalili, M. Zahar, M. Belbot, J. J. Kolata, K. Lamkin, D. J. Morrissey, B. M. Sherrill, M. Lewitowicz, and A. H. Wuosmaa, Phys. Rev. C 56, R2929 (1997).

[15] R. C. Johnson and P. J. R. Soper, Phys. Rev. 1, 976 (1970).

[16] M. Kamimura, M. Yahiro, Y. Iseri, H. Kameyama, Y. Sakuragi, and M. Kawai, Prog. Theor. Phys. Suppl. 89, 1
(1986).

[17] N. Austern, Y. Iseri, M. Kamimura, M. Kawai, G. Rawitscher, and M. Yahiro, Phys. Rep. 154, 125 (1987).

[18] J. S. Al-Khalili, J. A. Tostevin, and J. M. Brooke, Phys. Rev. C 55, R1018 (1997).

[19] J. A. Tostevin, J. S. Al-Khalili, J. M. Brooke, and J. A. Christley, J. Phys. G 24, 1589 (1998).

[20] J. M. Brooke, J. S. Al-Khalili, and J. A. Tostevin, Phys. Rev. C 59, 1560 (1999).

[21] H. Feshbach, in Interaction of High Energy Particles with Nuclei, edited by T.E.O. Ericson (Academic, New York 1967).

[22] H. Feshbach, Theoretical Nuclear Physics: Nuclear Reactions (Wiley, New York, 1992).

[23] H. Feshbach, in In Honor of Philip M. Morse, edited by H. Feshbach and U. Ingard (MIT, Cambridge, MA, 1969).

[24] M. V. Zhukov, A. A. Korsheninnikov, and M. H. Smedberg, Phys. Rev. C 50, R1 (1994).

[25] S. J. Wallace, Phys. Rev. C 12, 179 (1977).

[26] L. I. Schiff, Phys. Rev. 103, 443 (1956).

[27] D. S. Saxon and L. I. Schiff, Nuovo Cimento 6, 614 (1957).

[28] M. Rosen and D. R. Yennie, J. Math. Phys. 5, 1505 (1964).

[29] S. J. Wallace, Phys. Rev. D 8, 1846 (1973).

[30] S. J. Wallace, Phys. Rev. Lett. 27, 622 (1971).

[31] S. J. Wallace, Phys. Rev. D 9, 406 (1974).

[32] J. M. Brooke, Ph.D. thesis, University of Surrey, 1999.

[33] J. A. Christley, J. S. Al-Khalili, J. A. Tostevin, and R. C. Johnson, Nucl. Phys. A624, 275 (1997). 